Résumés des conférences et travaux

\title{
Histoire et archéologie de la Gaule romaine
}

\section{William Van Andringa}

\section{OpenEdition \\ Journals}

\section{Édition électronique}

URL : https://journals.openedition.org/ashp/3752

DOI : $10.4000 /$ ashp.3752

ISSN : 1969-6310

Éditeur

Publications de l'École Pratique des Hautes Études

\section{Édition imprimée}

Date de publication : 1 septembre 2020

Pagination : 168-177

ISSN : 0766-0677

Référence électronique

William Van Andringa, " Histoire et archéologie de la Gaule romaine ", Annuaire de l'École pratique des hautes études (EPHE), Section des sciences historiques et philologiques [En ligne], 151 | 2020, mis en ligne le 09 juillet 2020, consulté le 06 juillet 2021. URL : http://journals.openedition.org/ashp/3752 ; DOI : https://doi.org/10.4000/ashp.3752

Ce document a été généré automatiquement le 6 juillet 2021.

Tous droits réservés : EPHE 


\title{
Histoire et archéologie de la Gaule romaine
}

\author{
William Van Andringa
}

\section{Monuments funéraires, pouvoir des élites et mémoires familiales en Gaule (ler-IVe siècle)}

1 Lorsqu'il s'agit de définir les structures funéraires romaines, le premier problème rencontré est le caractère inopérant de notre vocabulaire actuel. Il n'y avait pas en effet de mot pour désigner les cimetières qui n'existent pas comme des ensembles polarisés et clos avant le Moyen Âge ${ }^{1}$. On parlait alors au singulier ou au pluriel de tombeaux, de sépultures ou d'aires funéraires (monumentum ou monimentum, sepulcrum, locus sepulturae, etc.). Le terme le plus courant est celui de monumentum qui désigne littéralement les monuments funéraires et dont la fonction était de garantir la pérennité des lignées familiales : monumentum est, quod memoriae servandae gratia existat. Un monument est quelque chose qui existe pour préserver la mémoire (Dig. XI, 7, 2, 6) ; Monumentum generaliter res est memoriae causa in posterum prodita (...). En général, un monument est quelque chose de montré et de transmis à la postérité pour la mémoire (Dig. XI, 7, 42). Parfois le monumentum est assimilable à un mausolée, c'est-à-dire à un grand monument patricien, comme à Nîmes (CIL XII, 3619): hoc monimentum maesoleumque, ce monument et mausolée, construit et dédié aux Mânes par Hostilia, fille de Lucius. On parlait aussi d'aires destinées aux sépultures, par exemple toujours à Nîmes (CIL XII, 4042): loc(us) sep(ulturae) p(edes) q(uadrati) XXV, emplacement des sépultures (qui composent le lot funéraire): 25 pieds carrés. Le plus souvent le monument funéraire n'est pas mentionné sur l'inscription: l'édicule, le podium, l'édifice est dédié au défunt dont il célèbre la mémoire ou aux dieux mânes qui le représentent. Ces tombes et tombeaux sont en principe regroupés ou alignés pour constituer des nécropoles, ce qui légitime l'emploi en archéologie du terme "ensemble funéraire " pour désigner des groupements de tombes, l'élément structurant de ces ensembles étant généralement l'axe viaire. À l'époque romaine, on installe les morts 
dans des secteurs en principe implantés le long des routes, d'accès à la ville pour les nécropoles suburbaines, qui sillonnent le territoire pour les tombeaux installés à la campagne.

2 La règle essentielle était en effet avant tout d'établir les morts dans des endroits fréquentés, parce que la posture sociale avait besoin, pour s'exprimer, des espaces publics, autrement dit du domaine viaire. Le défunt était d'abord un être social dont la légitimité civique s'affirmait par un monument dressé dans la périphérie urbaine. En effet, le faubourg qu'il soit désigné par le mot de suburbium, ce qui est au pied de la ville, ou par le terme de continentia, ce qui est attenant, en continuité avec l'espace urbain, était particulièrement adapté à exposer la mémoire civique de la communauté représentée par les tombeaux familiaux. Ce qui explique la grande variété des architectures des monuments funéraires ${ }^{2}$ : la diversité des formes adoptées participait tout simplement à la distinction nécessaire des mémoires familiales. Le système de la cité voulait donc que la mémoire des familles soit présentée en marge de ses lieux de vie. Parfois, dans l'expression des mémoires patriciennes, les deux se confondent, ainsi à Saint-Bertrand-de-Comminges (Lugdunum des Convènes) dans le cas du mausolée d'Herrane ${ }^{3}$. Les travaux récents menés dans la périphérie orientale de la ville ont montré que les vestiges d'un très grand tombeau $(13 \times 11 \mathrm{~m})$ étaient intégrés dans une grande enceinte délimitant un hortus associé à un grand bâtiment résidentiel très proche des limites urbaines. La proximité de la villa avec l'espace urbain (une centaine de mètres à peine) indique qu'elle constituait le domicile principal d'un personnage important qui pouvait à ce titre exercer une charge politique dans sa cité. Ce dispositif rappelle celui d'En Chaplix à Avenches / Aventicum : l'ensemble funéraire de l'époque julio-claudienne, composé d'un temple funéraire et de deux imposants mausolées, a été mis en relation une résidence suburbaine et à la grande famille helvète des Camilli ${ }^{4}$. Pour une époque plus tardive, le $\mathrm{IV}^{\mathrm{e}}$ siècle, les fouilles actuelles menées sur le site de Saint-Just à Saint-Bertrand-de-Comminges ont révélé les vestiges de trois mausolées monumentaux alignés le long d'un fossé parcellaire. En l'état du dossier, il est probable qu'il faille associer ces tombeaux familiaux à un complexe résidentiel proche situé à 500 mètres à peine de l'espace urbain ${ }^{5}$. Dans ce cas précis, la fondation de trois mausolées a certainement participé à l'établissement d'une villa comme centre de pouvoir nobiliaire à une époque, le $\mathrm{IV}^{\mathrm{e}}$ siècle, où la résidence urbaine ne s'imposait plus.

Pour les tombeaux situés à la campagne, la route jouait un rôle tout aussi central dans la visibilité des ensembles funéraires. À la Tourette, près de Lescar (PyrénéesAtlantiques), les monumenta (désignés "piles " par la tradition érudite) furent installés à $200 \mathrm{~m}$ de la villa, en bordure de route justement ${ }^{6}$. La situation est identique dans le cas de la villa trévire d'Echternach et de son mausolée. La mémoire familiale trouvait manifestement dans le domaine rural un lieu d'expression privilégié. Parfois celle-ci prenait appui sur une tombe plus ancienne comme à Saint-Herblain (Loire-Atlantique), chez les Namnètes ${ }^{7}$. À proximité d'une résidence rurale, un mausolée-temple fut construit à l'époque impériale, qui devait accueillir la tombe (ou le cénotaphe) du grand propriétaire du lieu. Le prestige de la mémoire familiale fut toutefois renforcé par le choix du lieu, occupé par un cairn du Bronze ancien volontairement intégré dans l'enceinte du mausolée. À proximité de celui-ci, la découverte de dix figurines moulées 
en terre blanche représentant Vénus, datées de la fin du $\mathrm{I}^{\mathrm{er}}$ et du début du $\mathrm{II}^{\mathrm{e}}$ siècle apr. J.-C., atteste de la célébration d'un culte funéraire peut-être à destination d'une matrone si l'on veut donner sens aux statuettes en question.

Dans la cité des Ausques, en Aquitaine méridionale, le monument-tour de Larroque semble indiquer un autre cas de figure ${ }^{8}$ (fig. 1 ).

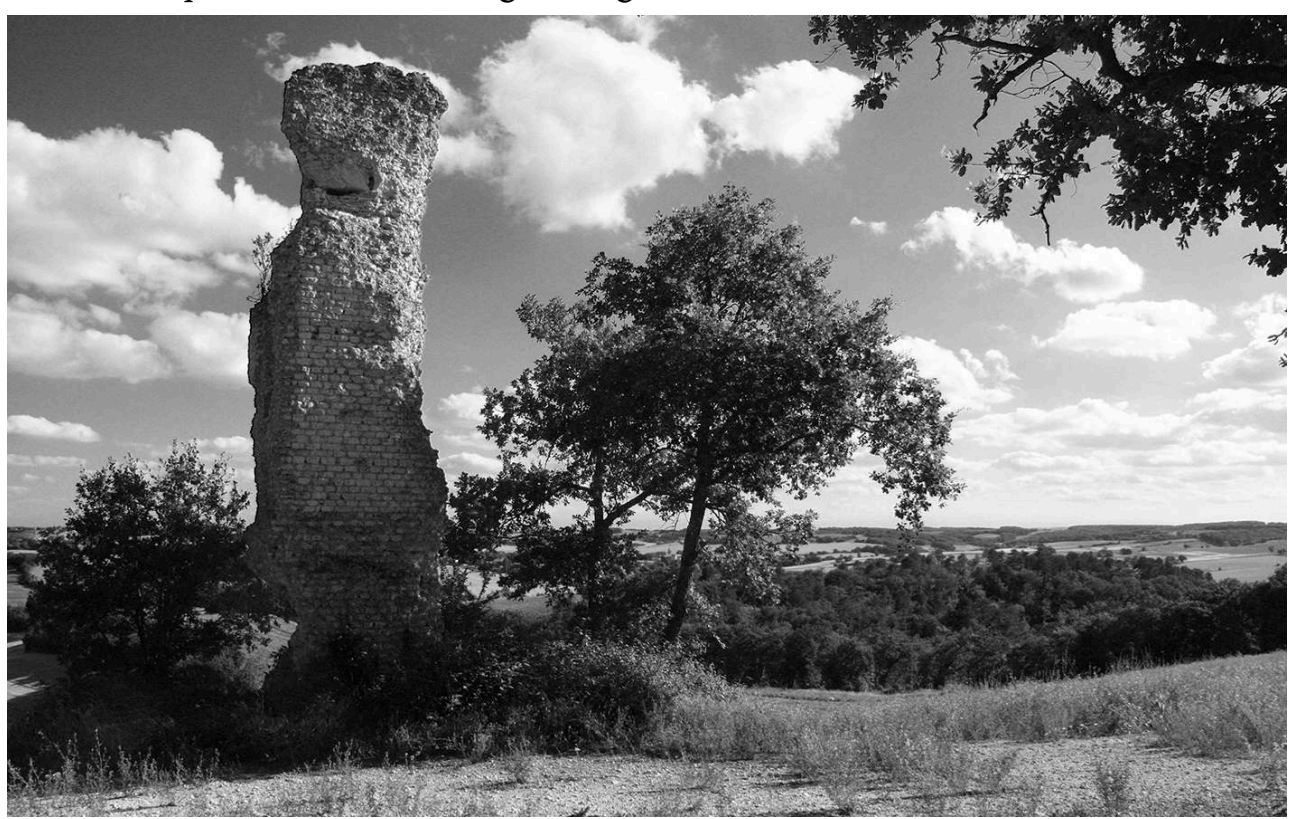

Fig. 1. - Le monument-tour de Larroque (Gers) installé au bord de la route romaine. La visibilité du tombeau dans le paysage exprime le pouvoir d'une famille sur un terroir de la cité des Ausques (cliché W. Van Andringa).

Le tombeau est encore en place le long de l'antique voie romaine qui reliait Eauze / Elusa à Auch / Augusta Auscorum. L'emplacement choisi pour le monument qui, visible sur des dizaines de kilomètres, surplombe le paysage environnant indique que la mémoire de la lignée familiale rayonnait sur un vaste territoire. Cet exemple est surtout révélateur du fait que les monuments funéraires constituaient des marquages forts des territoires, dans les vallées et sur les crêtes, le long des routes qui sillonnaient le territoire de la cité. Ce rôle de marqueur du paysage est pleinement exprimé chez les Agrimensores. Les monuments (monumenta) et les sépultures (sepulcra) marquaient des limites de parcelles et de terroirs; ils matérialisaient en ce sens l'ancrage des propriétés (par ex. dans les libri coloniarum, I, XII, 3 ; I, XIII, 5 ; II, I, 12 ; II, I, 17, etc. ; également Hygin II, 44). La prise en compte des tombeaux et d'autres éléments forts du paysage répondait au besoin d'assurer la cohésion des territoires à l'aide de repères visibles et pérennes. Dans les campagnes, les monuments funéraires, qui marquaient le pouvoir d'une famille sur un terroir, faisaient tout simplement autorité. Selon Frontin (II, 18), les tombeaux de certains personnages étaient reconnus comme des limites de territoires, ce qui n'empêchait pas (II, 44) que ceux-ci devaient demeurer malgré la division des terres. Le respect des monuments était assuré par la notoriété des titulaires. C'est tout le sens de l'heroum des Severii chez les Gabales, comme l'indique une inscription aujourd'hui disparue de Brugès (CIL XIII, 1571) : L. Seuer(ius) Seu[e]rus L. S(everi) N... f(ilius) o[m]nibus honori[b]us in ciuitate functus quiq(ue) hanc u[il]lam a solo instit[u]it et D(ecimus Seuerius) S(euerus) maior filior[um] heroum instituerunt pro salut [e] sua et suorum. Le pouvoir familial sur cette partie du territoire gabale fut affirmée par l'installation d'un cénotaphe consacré au fondateur de la villa. Il est probable que la 
tombe de celui-ci (définie par le dépôt de ses restes osseux) se trouvait dans la nécropole du chef-lieu, ce qui n'empêchait pas d'établir un mémorial dans le domaine rural familial. Cette autorité conférée aux tombeaux, attachée à la représentation foncière des notables, explique sans aucun doute leur préservation, ainsi certaines «piles » ont continué de servir de repères et de marqueurs du paysage au Moyen Âge et même jusqu'à nos jours (fig. 2).

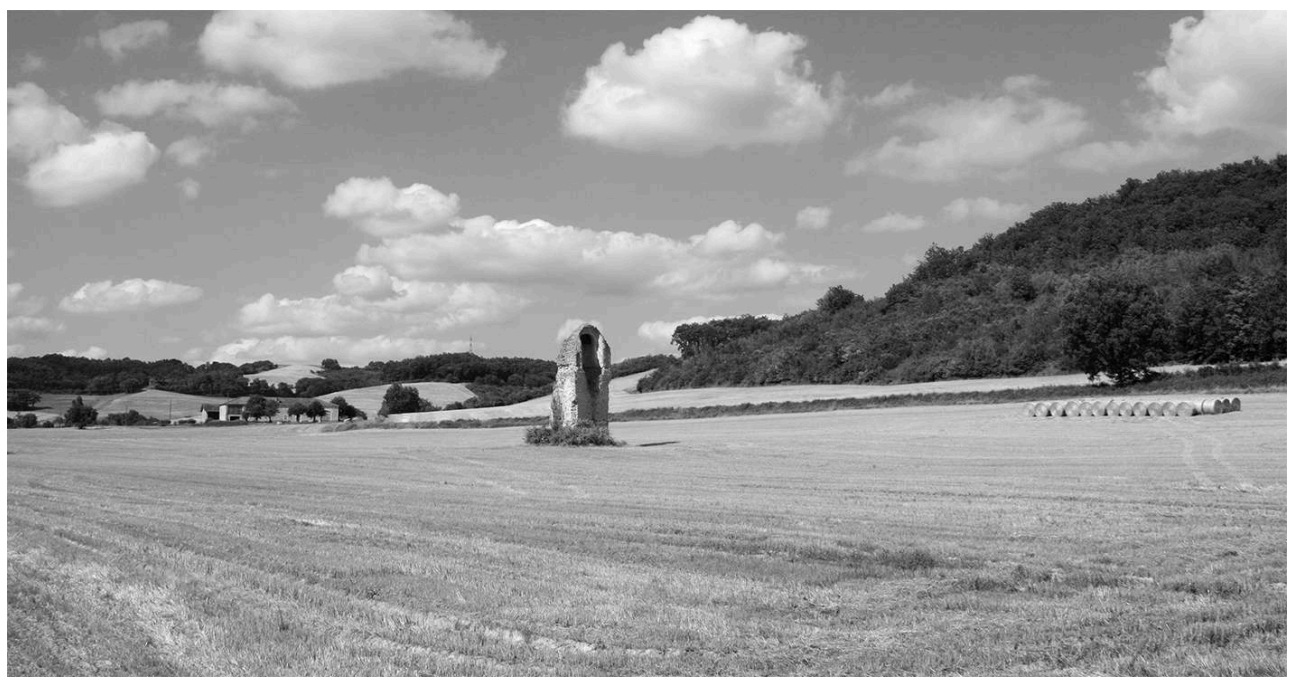

Fig. 2. - Le monument funéraire de La Turraque à Biran. Élément marquant du paysage et des propriétés foncières sur le temps long, les tombeaux constituaient de précieux repères topographiques pour les arpenteurs (cliché W. Van Andringa).

6 Ces exemples archéologiques amènent à explorer le lien entre les tombeaux, la mémoire d'un lieu et le pouvoir genticile. Comme l'a bien montré P. Geary pour le haut Moyen Âge, les élites possèdent un pouvoir fondamental qui est de dire la tradition. Ceux qui pouvaient contrôler le passé pouvaient commander l'avenir'. De telles politiques mémorielles pouvaient être instituées par le biais des tombeaux dont la fonction était justement de transmettre une mémoire familiale à la postérité. À Glanum, l'exemple est bien connu des Iulii de l'époque augustéenne qui ont légitimé leur pouvoir local et leur notoriété en construisant un mausolée à l'entrée de la ville, dédié aux parentes qui s'étaient illustrés dans les campagnes militaires de César ${ }^{10}$. La question reste débattue de la présence ou non des restes osseux des parents en question dans le monument, mais celle-ci est finalement secondaire, car un cénotaphe monumental avec statues et bas-reliefs montrant les hauts faits des défunts et dressé à l'entrée de la ville faisait tout autant l'affaire qu'un édifice défini par le dépôt effectif des restes osseux: la tombe définie par l'enterrement du mort et promise à l'oubli n'intervient finalement que de manière marginale dans le processus mémoriel. Construction politique, le tombeau pouvait être également inventé à partir d'un hautlieu ayant fixé la mémoire et les légendes locales. À Paule (Côte d'Armor), les fouilles d'Yves Menez ont révélé les vestiges monumentaux d'un puissant habitat gaulois installé au $\mathrm{VI}^{\mathrm{e}}$ siècle av. J.-C. Cette résidence aristocratique établie sur la longue durée fut démantelée à l'époque augustéenne, sans aucun doute en lien avec la fondation de la capitale des Osismes, Carhaix / Vorgium, à une dizaine de kilomètres de là ${ }^{11}$. Malgré cette destruction programmée, la mémoire familiale du lieu fut maintenue grâce à la reprise d'une nécropole de l'âge du Bronze qui bordait la résidence gauloise. Un enclos fut édifié autour de deux tumulus vénérables qui marquaient le paysage, provoquant la déviation de la vieille route. À l'intérieur, le tumulus oriental reçut un certain nombre 
d'aménagements qui s'expliquent par le choix d'un ancêtre et qui permettent d'identifier sans doute le tombeau attribué au fondateur mythique de la famille de Paule. Des monnaies autour de l'empreinte d'un élément en matière organique, probablement un tronc d'arbre, témoignent de rites spécifiques organisés au pied du tumulus. Des fours et des foyers ont également été mis au jour dans l'angle sud-est de l'enclos, près de deux bâtiments maçonnés. Ces structures ainsi que la découverte de nombreux fragments de figurines en terre blanche témoignent de l'installation d'un lieu de culte fréquenté jusqu'au $\mathrm{III}^{\mathrm{e}}$ siècle apr. J.-C. Sur la nature du culte, de deux choses l'une : soit les autorités responsables (de toute évidence le groupe familial qui s'identifiait au lieu) n'avaient pas connaissance du caractère funéraire de l'endroit, auquel cas ils attribuaient les tumuli à une intervention divine, dans le cadre d'un mythe local, soit ces autorités étaient conscientes du caractère sépulcral du lieu, auquel cas le culte célébré avait une connotation funéraire. Dans le premier cas, on peut penser à la recherche d'un espace mémoriel, reconnu par la mythologie locale. Dans l'autre, la valeur funéraire du lieu donnait clairement le sens au culte recherché. On sait qu'à Rome l'établissement d'un temple destiné à célébrer l'apothéose d'un personnage est attesté par Cicéron qui entend rendre un culte à sa fille défunte (Cic., Att. 12, 36). Il précise d'ailleurs qu'une telle fondation était possible à condition de construire le monument en question qualifié de fanum dans l'enceinte de sa villa. On pense également aux chapelles argiennes établies à Rome, toujours selon Cicéron, sur des lieux conservant les restes de défunts dont l'héroïsation avait évidemment un lien avec les origines de la cité. (Cic., De leg., 2, 55). On peut ainsi reconnaitre à Paule un lieu de culte établi sur un tombeau mythique, permettant de faire valoir l'ancestralité de la famille à une époque cruciale de restructuration des pouvoirs locaux après le recensement de 13-12 av. J.-C., alors qu'était planifiée la fondation de la ville capitale de Vorgium. L'appropriation mémorielle des tumuli de Paule a certainement joué un rôle dans la légitimation du pouvoir local des notables de Paule. Dans le même ordre idée, la mémoire de l'aristocrate gauloise de Goeblange-Nospelt qui reçut des funérailles dans la première moitié du $\mathrm{I}^{\mathrm{er}}$ siècle av. J.-C. fut entretenue pendant 150 ans après la fermeture de la tombe, comme en témoignent les pratiques rituelles observées dans l'enclos funéraire ${ }^{12}$. Là encore, il s'agissait vraisemblablement de légitimer le pouvoir d'une famille locale désormais intégrée dans la civitas Treverorum.

\section{Des traces aux gestes : pratiques funéraires de l'époque romaine}

7 Ce thème a été abordé dans le cadre de sept séances qui ont permis de présenter la documentation textuelle sur le sujet, mais surtout les données archéologiques provenant notamment de la fouille de la nécropole de Porta Nocera à Pompéi. Là, mieux qu'ailleurs, l'exceptionnelle préservation des nécro-sols et des structures funéraires permet d'étudier la mise en place et l'organisation d'une tradition funéraire locale. Des comparaisons avec la Gaule ont été proposées. Une conférence sur la méthodologie en criminalistique a permis de comparer les protocoles d'enregistrements utilisés par la police scientifique et par les archéologues.

8 Le 6 mars 2019, Nicolas Laubry (École française de Rome) a donné une conférence intitulée « Y a-t-il eu un droit des tombeaux en Gaule romaine ? Réflexions à partir des textes juridiques et épigraphiques. » Le 20 mars 2019, Henri Duday (PACEA, UMR 5199 du 
CNRS, Bordeaux) a donné une conférence intitulée « Le geste funéraire, vu du côté du défunt ». Le 3 avril 2019, Alice Dananai (Halma, UMR 8164 du CNRS, Lille) a donné une conférence intitulée «Gestes funéraires et pratiques locales. Le cas d'une microrégion des Atrébates (Artois-Ostrevant)». Le 10 avril 2019, Émilie Portat (Direction de l'archéologie à Chartres Métropole) a donné une conférence intitulée «Gestes funéraires à destination des tout-petits: l'exemple de la nécropole de Reverdy à Autricum / Chartres ». Le 17 avril 2019, Chloé Bouneau (Police technique et scientifique) a donné une conférence intitulée " De la trace au geste et à la preuve, méthodologie et interprétation en criminalistique ».

Dans un article de 1971, Lewis Binford s'attache à définir les approches des études sur les pratiques mortuaires, mettant en exergue les perspectives philosophiques sur les comportements humains face à la mort et les débats anthropologiques sur la question ${ }^{13}$. Il s'agissait, à cette époque, de mettre l'accent sur la mise en sens de mourir dans les sociétés humaines, de montrer l'impact des interprétations philosophiques et sociologiques, mais sans vraiment donner sa véritable importance à la mort archéologique, la mort telle qu'elle peut être décrite par un examen des vestiges et traces archéologiques. La raison était clairement, dans ces année-là, l'absence d'étude anthropologique poussée sur les gestes funéraires et surtout, sur beaucoup de chantiers archéologiques, l'absence de méthode susceptible de de révéler pratiques ou coutumes funéraires. Pour nos époques grecque et romaine, les funérailles de Patrocle ou les textes de loi de Cicéron continuaient de régir l'étude des coutumes funéraires des Anciens.

Presque 50 ans après, on peut dire que le développement récent de l'archéologie funéraire permet de renverser les perspectives. Depuis une trentaine d'années, l'étude des comportements funéraires dans les sociétés du passé a bénéficié d'apports méthodologiques majeurs. La taphonomie du cadavre et, plus largement, les diverses approches développées dans le cadre de l'archéothanatologie ont fourni aux spécialistes des questions funéraires des clés de lecture plus performantes pour comprendre l'organisation des pratiques mortuaires. L'enregistrement des traces et des dépôts autorise une restitution des gestes funéraires qui compose la pratique, des gestes techniques lorsqu'ils concernent la construction de la tombe ou l'aménagement du bûcher, des gestes constitutifs ou performatifs lorsqu'ils permettent de définir la structure funéraire, des gestes symboliques lorsqu'il s'agit d'organiser le changement de statut du défunt installé dans sa dernière demeure (rituels de passage) ou lorsqu'il s'agit d'arranger ou de mettre en scène la mémoire du défunt (rituels de mémoire), sans oublier les gestes involontaires ou non commandés par la pensée qui émaillent toute action humaine. De la trace au geste, l'archéologie permet d'étudier au plus près les comportements funéraires et même de comprendre leur variété. Ce réinvestissement technique du terrain, associé à une meilleure connaissance des matériaux (ossements humains et animaux, ossements ouvragés, végétaux, céramique, verre, etc.), ouvre des perspectives inespérées sur l'étude des pratiques funéraires, notamment sur la matérialité des gestes funéraires, sur l'impact des choses, artéfacts et écofacts, sur la 
pensée humaine ${ }^{14}$. Ce pouvoir des objets sur l'action funéraire est à mon sens perceptible dans les séquences rituelles restituées par la fouille de la nécropole de Porta Nocera à Pompéi ${ }^{15}$. Les traces étudiées montrent que les rites funéraires étaient structurés par une norme partagée par tous et fondée sur l'utilisation d'objets et de produits (parfum) ou aliments (fruits, viande) destinés à installer le défunt dans l'altérité radicale de la mort. Intervenaient également la tradition familiale (que l'on arrive à reconnaître par un travail spécifique sur chaque enclos) et l'initiative personnelle, certainement amplifiée par l'émotion en contexte funéraire, qui donnait finalement tout son sens au rituel célébré. Une fois la tombe constituée, certaines séquences étaient répétées, que l'on peut identifier à des rituels d'hommage et de mémoire en direction du défunt.

11 Cette enquête sur l'une des nécropoles romaines de Pompéi amène à insister sur l'importance dans la conduite des funérailles locales de quelques gestes élaborés à partir d'objets et de produits significatifs : une lampe à huile, un ou plusieurs flacons de parfum, une monnaie, un peu de viande ou quelques fruits prélevés dans la cuisine familiale suffisaient à construire l'altérité de la mort, à structurer les séquences rituelles, gage nécessaire de la bonne installation des défunts dans leur dernière demeure. Ces gestes sont à proprement parler les gestes rituels.

Le sens des rituels célébrés lors des funérailles résidait ainsi d'abord dans une liste précise d'objets et de produits ainsi que dans un enchaînement de gestes répétitifs qui faisaient ou réalisaient la cérémonie funéraire. La description des gestes que permet l'archéologie nous place sans doute au plus près de la signification des rites, parce que celle-ci réside pour une bonne partie dans les objets et les produits eux-mêmes ainsi que dans leur manipulation. Les objets déposés ou cassés, la lumière de la lampe, le parfum exhalé, les quelques aliments de qualité déposés sur le bûcher pouvaient certes être interprétés à la lumière de connaissances générales transmises par la culture romaine, écrite et orale (la purification, la transformation du défunt, son installation dans la tombe), mais la redondance des vestiges et des faits archéologiques montre que l'essentiel résidait dans le pouvoir intrinsèque de ces objets ou de ces produits, dans leur capacité à générer une expérience, un cadre sensuel, olfactif et émotionnel ainsi qu'évidemment dans leur manipulation à des moments différents permettant d'élaborer et d'orchestrer la cérémonie funèbre. Le sens était construit plutôt qu'il n'était donné.

13 Une partie du sens des funérailles résidait dans un jeu d'oppositions qui réalisaient le passage dans la mort; et ce jeu d'oppositions était créé non pas par la pensée ou une symbolique complexe, mais par la flamme et la matérialité de la lampe domestique, par l'odeur du parfum et la matérialité des flacons, par la nourriture emportée par le brasier du bûcher ; la mort était avant tout organisée autour du corps par des objets et des offrandes de même qu'elle était installée dans des structures mémorielles soigneusement hiérarchisées, la tombe pour les restes physiques de l'individu et le monument pour la mémoire sociale de la famille. L'emploi redondant et régulier de la lampe et du parfum rend compte de l'importance des expériences olfactives et visuelles dans la performance rituelle et dans sa transmission. La perception olfactive est, comme le note Joël Candau ${ }^{16}$, «fortement associée à l'encodage des souvenirs et à leur rappel ». Il est ainsi très probable que dans le contexte émotionnel fort des funérailles, la mémoire olfactive ait joué un rôle structurant dans l'élaboration et la transmission des savoir-faire rituels. La mise en scène ou le bris des flacons sanctionnait en quelque 
sorte la performance personnelle du rite, sous la forme de traces matérielles reconnaissables. susceptible d'être reconnue par tous, ne requérait pas la stricte observance de règles matérielles : une torche pouvait remplacer une lampe et le parfum n'être versé qu'une fois sans que le flacon ne soit brisé. À l'inverse, on pouvait prendre un grand soin, parce qu'on l'avait vu faire par un membre de la famille, à briser les flacons et en aligner les fragments devant la stèle, en donnant finalement autant d'importance à un geste personnel qu'à l'acte commun des profusiones. Cet enrichissement gestuel de l'archétype «offrande de parfum » complétait finalement le sens primaire du rite en lui donnant une envergure personnelle ou familiale. Ce qui comptait était bien de célébrer le souvenir du défunt et de marquer son passage par un geste personnel, témoignant de l'implication d'un proche. On parle bien de pratiques funéraires, résultant de la relation dialectique entre une situation et un habitus, entendu comme un système de dispositions durables et transposables, transmises par l'éducation. ainsi que leur rôle structurant des cérémonies funèbres participaient à l'élaboration d'une culture funéraire commune, partagée par un grand noticipaient à lélaboration croit les points communs relevés entre Pompéi et nombre de cités d'Italie ou de Narbonnaise. L'identité romaine résidait finalement dans la reconnaissance d'un corps de pratiques communes face à la mort que l'on peut résumer par l'usage de rituels d'opposition ou liés à la transformation du défunt, incarnés dans bien des lieux par les mêmes objets et produits de la vie courante, lampes, parfum, vin, viande de porc ou de poulet. La force de ce savoir commun était de pouvoir être incarné par des objets et des produits spécifiques, beaucoup plus que par des préceptes philosophiques ou religieux complexes sur l'au-delà.

\section{Actualité de la recherche : archéologie et société en France}

16 Une série de conférences a par ailleurs été l'occasion de présenter les institutions archéologiques françaises et de discuter de l'organisation de l'archéologie en France aujourd'hui. Un débat sur les enjeux institutionnels de l'archéologie contemporaine a été animé en compagnie de Frédérik Letterlé (UMR 5138, laboratoire ArAr, Lyon) et d'Hugues Bonnefon (Sciences-Po Paris). La question du grand écart actuel entre les moyens, humains et financiers, alloués à l'archéologie préventive et à l'archéologie programmée a également été discutée. Elle témoigne de la nécessité d'un rééquilibrage dans l'organisation de nos pratiques archéologiques.

Le 16 janvier 2019, Dominique Joly (ancien directeur du Service archéologique de Chartres) a donné une conférence intitulée « Du sauvetage à l'archéologie préventive : une histoire récente de l'archéologie à Chartres ». Le 23 janvier 2019, Hugues Bonnefon a donné une conférence intitulée «L'archéologie préventive en chantier. La fabrique des institutions ». Le 30 janvier 2019, Frédérik Letterlé a donné une conférence intitulée

Annuaire de l'École pratique des hautes études (EPHE), Section des sciences historiques et philologiques, 151 | 2020 
«Évolution juridique de l'archéologie et activité des services régionaux de l'archéologie ». Le 13 février 2019, Dominique Joly a donné une conférence intitulée « 10000 unités stratigraphiques: le site du Cinéma à Chartres ou l'histoire récente d'une fouille urbaine $»$.

Les dernières séances du séminaire ont concerné l'espace urbain, à travers deux questions, la première concernant l'aménagement des rues et le pouvoir des édiles, l'autre intéressant les aménagements religieux des gens de métier dans la fabrique de la ville : le 15 mai 2019, Anaïs Pinhède (service archéologique de Chartres) a donné une conférence intitulée «La rue et le pouvoir urbain à Chartres / Autricum. Réflexion sur l'évolution du système viaire d'une ville romaine». Le 22 mai 2019, Aude Durand (doctorante EPHE) a donné une conférence intitulée "Aménagements religieux des gens de métier et espaces urbains en Italie romaine».

\section{NOTES}

1. N. Laubry, «Les lieux funéraires dans la Rome ancienne: désignations et configurations (II s. av. n. è. - III ${ }^{\mathrm{e}}$ s. de n. è.)", dans M. Lauwers et A. Zemour (dir.), Qu'est-ce qu'une sépulture? Humanités et systèmes funéraires de la Préhistoire à nos jours, Actes des $36^{e}$ Rencontres internationales d'archéologie et d'histoire d'Antibes, 2015, Antibes, 2016, p. 75-94. Pour une définition archéologique des lieux funéraires, W. Van Andringa, "Le monument et la tombe. Deux façons de mourir à l'époque romaine ", dans M.-D. Nenna, S. Huber et W. Van Andringa (éd.), Constituer la tombe, honorer les défunts en Méditerranée antique, Alexandrie, Centre d'études alexandrines, 2018 (Études alexandrines, 46), p. 381-402. Pour la Gaule, M. Monteil, W. Van Andringa, «Hoc monimentum maesoleumque. Les monuments funéraires dans le paysage des cités des Gaules et des Germanies romaines ", dans Monumentum fecit. Monuments funéraires en Gaule romaine, Gallia, 76-1 (2019), p. 1-8.

2. P. Gros, L'architecture romaine 2, maisons, palais, villas et tombeaux, Paris, Picard, 2001 ; Id., « Aspects sociaux et monumentaux des alignements funéraires à l'entrée des villes romaines ", dans F. Michaud-Fréjaville, N. Dauphin, J.-P. Guilhembet (dir.), Entrer en ville, Rennes, Presses universitaires de Rennes, 2006, p. 39-52.

3. W. Van Andringa et al., «Face à la ville : le mausolée d'Herrane à Saint-Bertrand-deComminges / Lugdunum des Convènes (Haute-Garonne) », Gallia, 76-1 (2019), p. 55-70.

4. L. Flutsch, P. Hauser (dir.), Le mausolée nouveau est arrivé! Les monuments funéraires d'Avenches-en-Chaplix, 2 vol., Lausanne, 2012 (Cahiers d'archéologie romande).

5. W. Van Andringa (dir.), Saint-Bertrand-de-Comminges / Valcabrère: entre ville et campagne. Archéologie d'une périphérie urbaine de l'époque romaine au Moyen Âge, rapport du Projet collectif de recherches, 2019, p. 29-338. 
6. P. Clauss-Balty (dir.), Les piles funéraires gallo-romaines du Sud-Ouest de la France, Pau, Presses universitaires de Pau et des Pays de l'Adour, 2016.

7. F. Mercier et al., «Fondation d'une mémoire familiale dans la cité des Namnètes : le mausolée antique des Pellières à Saint-Herblain (Loire-Atlantique) », Gallia, 76-1, 2019, p. 213-225.

8. P. Clauss-Balty, Les piles funéraires gallo-romaines du Sud-Ouest.

9. P. Geary, La mémoire et l'oubli à la fin du premier millénaire, Paris, 1996.

10. P. Gros, « Le mausolée des Iulii et le statut de Glanum », Revue archéologique, 1 (1981), p. 65-80.

11. Y. Menez, Une résidence de la noblesse gauloise: le camp de Saint-Symphorien à Paule (Côtes-d'Armor), Paris, MSH (Documents d'archéologie française), à paraître.

12. J. Metzler, C. Gaeng et al., Goeblange-Nospelt. Une nécropole aristocratique trévire, Luxembourg, 2009 (Dossiers d'archéologie du Musée national d'histoire et d'art, XIII).

13. L. Binford, "Mortuary Practices: Their Study and Their Potential», Memoirs of the Society for American Archaeology, 25 (1971), p. 6-29.

14. W. Van Andringa, "Rituels pour les morts et culte des dieux à Pompéi », dans J. Jouanna, A. Vauchez, J. Scheid et M. Zink (éd.), Des tombeaux et des dieux. Actes du colloque de la villa Kérylos, Paris, 2019 (Cahiers de la villa Kérylos, $\mathrm{n}^{\circ}$ 30), p. 99-119. Selon Nicole Boivin, Material Cultures, Material Minds. The impact of things on Human Thought, Society, and Evolution, Cambridge, Cambridge University Press, 2008, la manipulation d'objets peut faire le sens d'une action, sans qu'il soit nécessaire de donner une raison à l'utilisation de l'objet ou d'être en mesure d'exprimer un sens abstrait pour tout type de pratique.

15. W. Van Andringa, H. Duday, S. Lepetz et al., Mourir à Pompéi. Fouille d'un quartier funéraire de la nécropole romaine de Porta Nocera (2003-2007), 2 vol., Rome, 2013 (Collection de l'École française de Rome, 468).

16. J. Candau, Mémoires et expériences olfactives. Anthropologie d'un savoir-faire sensoriel, Paris, 2000, p. 84-85.

\section{RÉSUMÉS}

Programme de l'année 2018-2019: I. Monuments funéraires, pouvoir des élites et mémoires familiales en Gaule (Ir-IV siècle apr.J.-C.). - II. Des traces aux gestes : pratiques funéraires de l'époque romaine. III. Actualité de la recherche : archéologie et société en France.

\section{INDEX}

Thèmes : Histoire et archéologie de la Gaule romaine 


\section{AUTEUR}

\section{WILLIAM VAN ANDRINGA}

Directeur d'études, École pratique des hautes études - section des Sciences historiques et philologiques 